ARTICLE

DOI: $10.1038 / s 41467-018-07123-y$

\title{
Signature of quantum Griffiths singularity state in a layered quasi-one-dimensional superconductor
}

\author{
Enze Zhang ${ }^{1,2}$, Jinhua Zhi ${ }^{3}$, Yi-Chao Zou ${ }^{4,5}$, Zefang $\mathrm{Ye}^{1,2}$, Linfeng $A i^{1,2}$, Jiacheng Shi, ${ }^{1,2}$, Ce Huang ${ }^{1,2}$, \\ Shanshan Liu'1,2, Zehao Lin ${ }^{1,2}$, Xinyuan Zheng ${ }^{1,2}$, Ning Kang ${ }^{3}$, Hongqi Xü ${ }^{3}$, Wei Wang ${ }^{6}$, Liang He ${ }^{6}$, Jin Zou (i) ${ }^{4,5}$, \\ Jinyu Liu ${ }^{7}$, Zhiqiang Mao ${ }^{7} \&$ Faxian Xiu ${ }^{1,2,8}$
}

Quantum Griffiths singularity was theoretically proposed to interpret the phenomenon of divergent dynamical exponent in quantum phase transitions. It has been discovered experimentally in three-dimensional (3D) magnetic metal systems and two-dimensional (2D) superconductors. But, whether this state exists in lower dimensional systems remains elusive. Here, we report the signature of quantum Griffiths singularity state in quasi-one-dimensional (1D) $\mathrm{Ta}_{2} \mathrm{PdS}_{5}$ nanowires. The superconducting critical field shows a strong anisotropic behavior and a violation of the Pauli limit in a parallel magnetic field configuration. Currentvoltage measurements exhibit hysteresis loops and a series of multiple voltage steps in transition to the normal state, indicating a quasi-1D nature of the superconductivity. Surprisingly, the nanowire undergoes a superconductor-metal transition when the magnetic field increases. Upon approaching the zero-temperature quantum critical point, the system uncovers the signature of the quantum Griffiths singularity state arising from enhanced quenched disorders, where the dynamical critical exponent becomes diverging rather than being constant.

\footnotetext{
${ }^{1}$ State Key Laboratory of Surface Physics and Department of Physics, Fudan University, 200433 Shanghai, China. ${ }^{2}$ Institute for Nanoelectronic Devices and Quantum Computing, Fudan University, 200433 Shanghai, China. ${ }^{3}$ Bejing Key Laboratory of Quantum Devices, Key Laboratory for the Physics and Chemistry of Nanodevices and Department of Electronics, Peking University, 100871 Beijing, China. ${ }^{4}$ Materials Engineering, The University of Queensland, Brisbane, QLD 4072, Australia. ${ }^{5}$ Centre for Microscopy and Microanalysis, The University of Queensland, Brisbane, QLD 4072, Australia. ${ }^{6}$ School of Electronics Science and Engineering, Nanjing University, 210093 Nanjing, China. ${ }^{7}$ Department of Physics and Engineering Physics, Tulane University, New Orleans, LA 70118, USA. ${ }^{8}$ Collaborative Innovation Center of Advanced Microstructures, Nanjing University, 210093 Nanjing, China. Correspondence and requests for materials should be addressed to F.X. (email: Faxian@fudan.edu.cn)
} 
S uperconductivity in materials with low-dimensional electronic structure becomes an important topic during the past decades because they provide a rich avenue for investigating exotic physical properties and their potential applications in quantum computing devices ${ }^{1,2}$. With the reduction of the dimensionality, fluctuation, disorder, and quantum correlation effects begin to have a special influence on the superconducting characteristics $^{3}$. As a result, many interesting phenomena arise, such as localization of Cooper pairs ${ }^{4}$, transition temperature oscillations ${ }^{5,6}$, and quantum phase transition (QPT) at zero temperature $^{7-9}$. In particular, one example of the QPT has been extensively studied that is the superconductor-insulator transition (SIT) or superconducting-metal transition (SMT), in which a continuous phase transition occurs at the zero-temperature limit as a function of external tuning parameters, such as external electric fields, carrier density, and out-of-plane magnetic fields ${ }^{1,9}$. However, despite numbers of investigations in various systems, there still remain many open issues, such as unexplained inconsistencies among the critical exponents found in different physical systems ${ }^{1,10}$ and the appearance of intervening quantum metallic state between the superconducting and the insulating state $^{2,11}$. The latest remarkable observations of the quantum Griffiths singularity in thin Ga films ${ }^{12}, \mathrm{LaAlO}_{3} / \mathrm{SrTiO}_{3}(110)$ interface ${ }^{13}$, monolayer $\mathrm{NbSe}_{2}{ }^{14}$, and ionic liquid gated $\mathrm{ZrNCl}$ and $\mathrm{MoS}_{2}{ }^{15}$ shed a new light on the understanding of SMT in two-dimensional (2D) system $^{16}$. Whether this phenomenon would appear in lower dimensional superconducting systems remains elusive.

Very recently, the newly discovered quasi-one-dimensional (1D) transition metal chalcogenide superconductors with a formula $\mathrm{M}_{2} \mathrm{Pd}_{\mathrm{x}} \mathrm{Q}_{5}(\mathrm{M}=\mathrm{Ta}, \mathrm{Nb} ; \mathrm{Q}=\mathrm{S}$, Se $)$ have attracted great attention because of their exotic superconducting characteristics ${ }^{17,18}$. The most striking feature of these compounds is their remarkably large upper critical fields, which exceed the Pauli limit relative to their transition temperature ${ }^{19,20}$. Theoretical investigations show that this should be due to the combination of strong spin-orbit coupling and multiband effects in the extreme dirty limit ${ }^{21}$. More interestingly, instead of using the sputtering or evaporating technique in fabricating granular and amorphous nanowires, the layered low-dimensional nature in such compounds offers a wonderful platform for investigating SMT in quasi-1D single-crystal nanowires ${ }^{17}$.

In this study, we report the systematic transport measurements on quasi-1D $\mathrm{Ta}_{2} \mathrm{PdS}_{5}$ nanowires obtained by Scotch tape assisted micromechanical exfoliation of bulk crystals. The critical field of the system shows a strong anisotropic behavior and a violation of Pauli paramagnetic law under parallel magnetic field configuration. Moreover, the current-voltage $(I-V)$ characteristics of the nanowires in the superconducting regime display a series of multiple voltage steps resulting from quantum phase slip; and a hysteresis arises when the applied current is swept up and down, which resembles a typical quasi-1D behavior in superconductivity. Interestingly, the magnetotransport properties of the nanowires undergo a SMT with increasing magnetic field. As the temperature approaches zero, the dynamical critical exponent shows a divergent value, which is a signature of quantum Griffiths singularity state.

\section{Results}

Sample characterization. $\mathrm{Ta}_{2} \mathrm{PdS}_{5}$ single crystals were synthesized using chemical vapor transport (CVT) method ${ }^{22}$ (see Methods for details). The as-grown bulk crystals exhibit a long needle-like structure with a length up to centimeters. As shown in Fig. 1a, $\mathrm{Ta}_{2} \mathrm{PdS}_{5}$ is composed of corrugated metal sulfide sheets, which form $1 \mathrm{D}$ chains along the $<010>$ direction (needle direction or $b$-axis) ${ }^{19,21}$. Its crystal structure is centrosymmetric, with the space group of $\mathrm{C} 2 / \mathrm{m}$. Due to its low-dimensional structure, the $\mathrm{Ta}_{2} \mathrm{PdS}_{5}$ bulk material can be cleaved into rectangular-shape nanowires where the width ranges from 0.1 to $2 \mu \mathrm{m}$, with the thickness of 70-300 nm. Figure $1 \mathrm{~b}$ shows the scanning electron microscope (SEM) image of the exfoliated nanowires on polydimethylsiloxane substrate, where the long axis of the nanowire is the $b$-axis of the crystal. Figure 1c shows the bright-field transmission electron microscopy (TEM) image taken from a $\mathrm{Ta}_{2} \mathrm{PdS}_{5}$ nanowire. Figures $1 \mathrm{~d}$, e respectively show the corresponding selective-area electron diffraction (SAED) pattern and high-resolution TEM (HRTEM) image, which confirm that the axial direction of the $\mathrm{Ta}_{2} \mathrm{PdS}_{5}$ nanowire is $<010>$ and the single-crystal nature of the nanowire. Figures $1 \mathrm{f}-\mathrm{h}$ show the elemental maps taken from a section of the nanowire (yellow dashed square in Fig. 1c) by energy-dispersive X-ray spectroscopy (EDS), indicating that $\mathrm{Ta}, \mathrm{Pd}$, and $\mathrm{S}$ are distributed evenly in the nanowire.

Anisotropic superconductivity in $\mathrm{Ta}_{2} \mathrm{PdS}_{5}$ nanowires. Fourterminal $\mathrm{Ta}_{2} \mathrm{PdS}_{5}$ nanowire devices with various thickness were fabricated using $e$-beam lithography (EBL), followed by a metal deposition process (See Methods for details). A typical device structure is schematically illustrated in Fig. 2a (Optical image, inset of Fig. 2b). The measured four-probe resistance is defined as $R=V_{\mathrm{XX}} / I_{\mathrm{DS}}$, where $I_{\mathrm{DS}}$ is the source-drain current and $V_{\mathrm{XX}}$ is the measured voltage drop between the middle two voltage probes. As depicted in Fig. 2b, four-terminal temperature-dependent resistance of the nanowire device shows a metallic behavior upon cooling and the superconductivity appears at $T_{\mathrm{C}} \sim 3.3 \mathrm{~K}$ (Device 01 cross-sectional area, $120 \mathrm{~nm}$ (thickness) $\times 300 \mathrm{~nm}$ (width)), where $T_{\mathrm{C}}$ is defined as the temperature corresponding to the middle point of the superconducting transition. $T_{\mathrm{C}}$ values of devices with different thickness are shown in Supplementary Figure 3 in which $T_{\mathrm{C}}$ decreases monotonically with the thickness. Note that in order to control the variables, we intentionally compare the samples with a similar channel width. We also measure the $T_{\mathrm{C}}$ of different lengths in one nanowire (Supplementary Figure 3) and different parts of the nanowire exhibit a similar $T_{\mathrm{C}}$, indicative of good uniformity ${ }^{17}$.

Next, we explore the anisotropy of the superconductivity in $\mathrm{Ta}_{2} \mathrm{PdS}_{5}$. Figures $2 \mathrm{c}$, e display the temperature-dependent resistance for magnetic fields applied perpendicular and parallel to the $b$-axis of $\mathrm{Ta}_{2} \mathrm{PdS}_{5}$ nanowire device (inset Fig. 2d), respectively. In the case of the perpendicular geometry, the superconductivity rapidly disappears with the increase of magnetic field and is completely quenched when the magnetic field is larger than $6.5 \mathrm{~T}$. For the parallel scenario, however, the superconductivity is robust against the magnetic field and it survives even under $9 \mathrm{~T}$ at $T=2 \mathrm{~K}$. Figure $2 \mathrm{~d}$ shows the angledependent upper critical field $B_{\mathrm{C} 2}(T)$ at $T=2.4 \mathrm{~K}$ (similar device to device 01 , the data of device 01 are shown in Supplementary Figure 4), where it exhibits large anisotropy. We define $B_{\mathrm{C} 2}(T)$ as the magnetic field where the resistance drops to $50 \%$ of the normal resistance. We tried to fit the angular-dependent $B_{\mathrm{C} 2}(T)$ at small $\theta$ values using both $3 \mathrm{D}^{23,24}$ and $2 \mathrm{D}^{25,26}$ models, where neither of them fits the data well (See Supplementary Figure 6). Then, we further measured the angle-dependent $B_{\mathrm{C} 2}(T)$ of $\mathrm{Ta}_{2} \mathrm{PdS}_{5}$ samples with different thickness (Supplementary Figure 7). We find that the angle-dependent $B_{\mathrm{C} 2} \sim 5 \mu \mathrm{m}$ (Bulk) sample shows a very good fit to the $3 \mathrm{D}$ model, which is consistent with the previous study ${ }^{27}$. However, the angle-dependent $B_{\mathrm{C} 2}$ of thin nanowire samples do not fit well with either 3D or 2D model, indicating the reduced dimensionality of its superconductivity (See Supplementary Note 2 for details). As far as we know, up to 
a
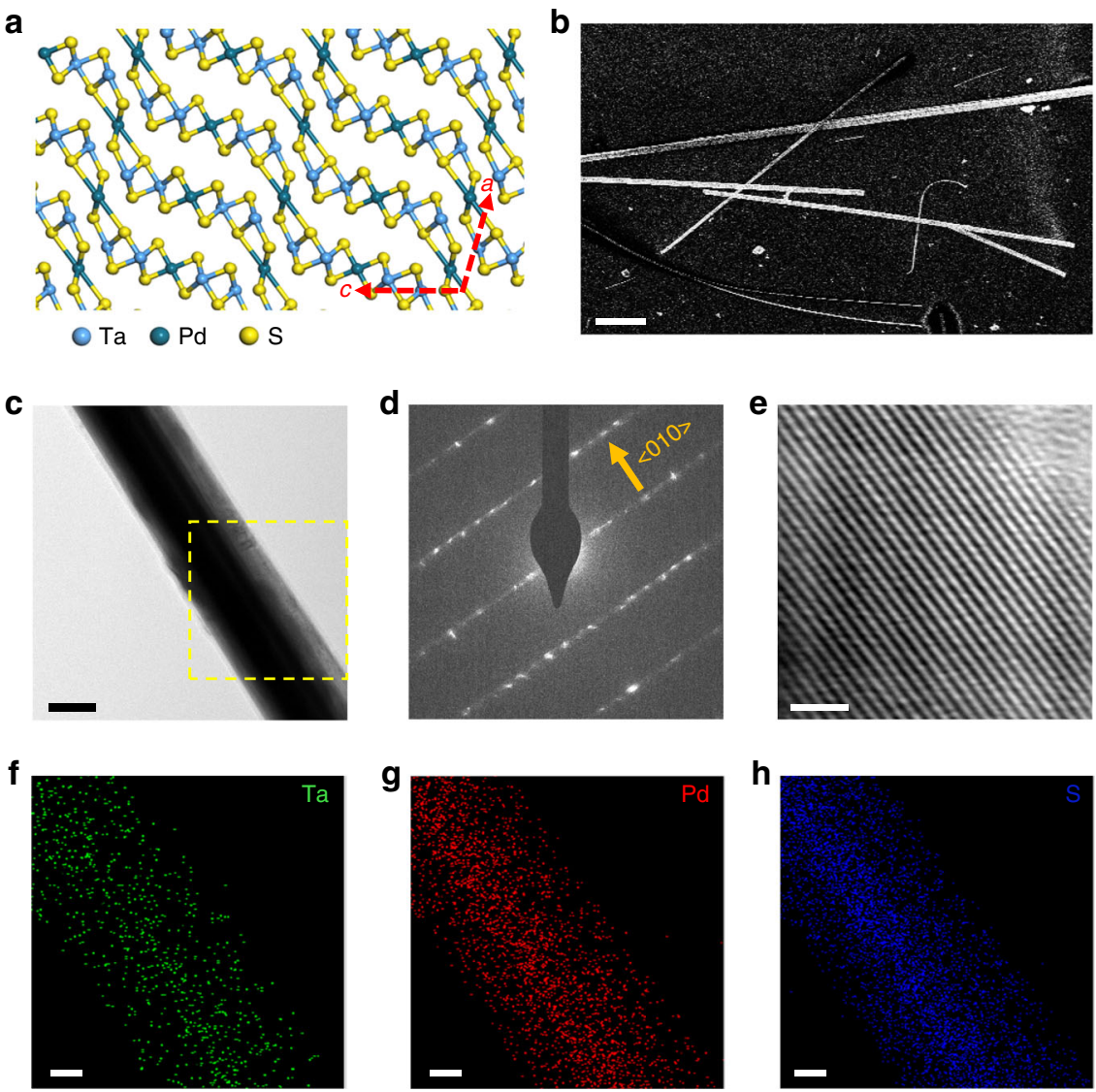

Fig. 1 Crystal structure and characterizations of $\mathrm{Ta}_{2} \mathrm{PdS}_{5}$ crystals. a Crystal structure of $\mathrm{Ta}_{2} \mathrm{PdS}_{5}$ viewed along the $b$-axis, exhibiting layered structure in the plane perpendicular to $a$-axis of the crystal. $\mathbf{b} \mathrm{SEM}$ image of the exfoliated $\mathrm{Ta}_{2} \mathrm{PdS}_{5}$ nanowire. Scale bar, $5 \mu \mathrm{m}$. c Bright-field TEM image taken from a section of a typical $\mathrm{Ta}_{2} \mathrm{PdS}_{5}$ nanowire. Scale bar, $100 \mathrm{~nm}$. d Corresponding SAED pattern showing that the axial growth direction of the nanowire is $<010>$. e Corresponding HRTEM. Scale bar, $100 \mathrm{~nm}$. f-h EDS elemental maps taken from the region marked by a square in $\mathbf{c}$, for Ta (f), Pd (g), and S (h). Scale bars, $50 \mathrm{~nm}$

now, there is no theoretical models/equations that can be used for the data fitting in the quasi-1D superconducting system, which calls for a further theoretical investigation. In addition, the dependence of $B_{\mathrm{C} 2}$ on temperature $T$ for both the parallel and perpendicular magnetic fields are fitted well using the empirical equation $^{17}$ :

$$
B_{\mathrm{C} 2}(T)=B_{\mathrm{C} 2}(0)\left(1-\frac{T}{T_{\mathrm{C}}}\right)^{2},
$$

where $B_{\mathrm{C} 2}(0)=\Phi_{0} / 2 \pi \xi(0)^{2}$. Then, the Ginsburg-Landau (GL) coherence length for parallel and perpendicular configuration can be estimated to be $\xi_{/ /}(0)=4.1 \mathrm{~nm}$ and $\xi_{\perp}(0)=7.6 \mathrm{~nm}$, respectively. Considering the relatively small anisotropy in the $a-c$ plane (See Supplementary Note 3 for details), the coherence along $a$ - and $c$-axis should be around $4.1 \mathrm{~nm}$. Note that for the parallel configuration, the upper critical field at $T=0 \mathrm{~K}$ seemingly exceeds the Pauli paramagnetic limit of weak coupling Bardeen-Cooper-Schrieffer (BCS) superconductors $B_{\mathrm{P}} \mathrm{BCS}=\Delta_{0} /$ $\sqrt{2} k_{\mathrm{B}} T_{\mathrm{C}}=1.84 T_{\mathrm{C}}=6.1 \mathrm{~T}$ (dashed line in Fig. $2 \mathrm{f}$ ). Compare with spin-momentum lock induced Ising superconductivity in monolayer $\mathrm{NbSe}_{2}{ }^{14,28}$ and ionic liquid gated $\mathrm{MoS}_{2}{ }^{26}$, the violation of Pauli paramagnetic limit in $\mathrm{Ta}_{2} \mathrm{PdS}_{5}$ is resulted from the synergetic effect of strong spin-orbit coupling and multiband effects $^{21,29}$.

I- $V$ characteristics of $\mathrm{Ta}_{2} \mathrm{PdS}_{5}$ nanowire devices and the estimation of penetration depth. In order to further understand the superconductivity in $\mathrm{Ta}_{2} \mathrm{PdS}_{5}$ nanowires, we performed fourterminal current-voltage $(I-V)$ measurements in the superconducting transition region. Figure 3 shows the measured $I$ - $V$ curves at varying temperatures (Fig. 3a) and magnetic fields (Fig. 3b) on a linear scale driven by current. A series of sharp voltage steps were observed as the nanowires transit from the superconducting to the normal state. These voltage steps are reproducible, becoming more pronounced and sharper at lower temperatures (magnetic fields) and finally vanished with the gradual increase of temperature (magnetic field). Usually, the multiple voltage steps in the $I-V$ characteristics are considered to be typical features of quasi-1D superconductors. It has been reported in thin superconducting polycrystalline $\mathrm{Nb}$ nanowires $^{30,31}$ and single-crystal Sn nanowires ${ }^{32}$. These voltage steps were interpreted as a consequence of spatially localized weak spots or resistive phase slip centers (PSCs), which arise from the local imperfections or defects in the nanowire that support a smaller critical current. A voltage step in the wire is created when the applied excitation current exceeds the local critical current of a specific PSC. The spatial extension of the PSC is typically on the order of a few micrometers in length ${ }^{32}$, which is at the same level of our device length. In addition, when we sweep the current upstream and back down, a sequence of hysteresis loop has been observed in the superconducting regime (Figs. $3 \mathrm{c}$, d), which also disappears as the temperature or magnetic field increases. As the phase-slip centers in superconducting nanowires act qualitatively like weak-link in Josephson junctions, a superconducting nanowire can be viewed as the coupled combination of Josephson junctions and the rest of the superconducting filament ${ }^{33}$. Hence, such a hysteresis in $I-V$ 
a

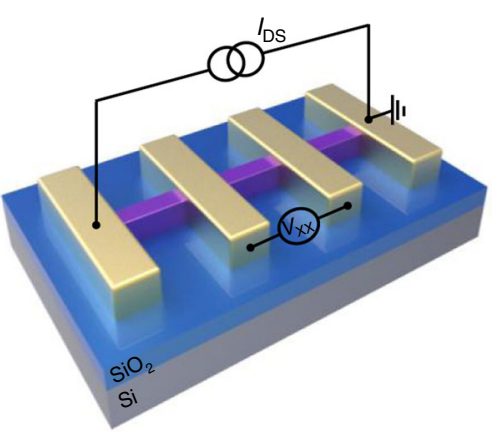

C

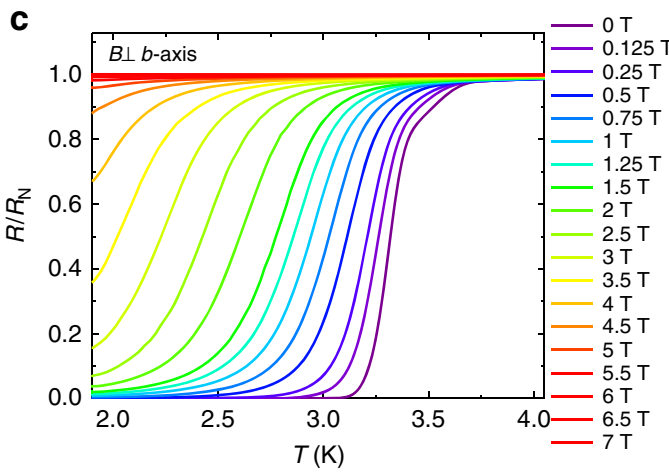

e

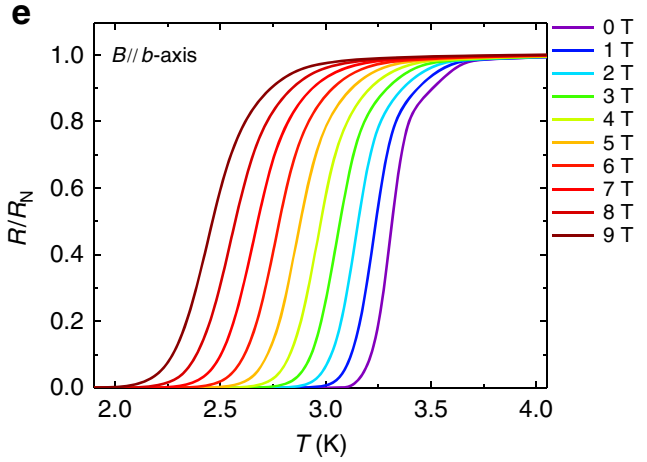

b

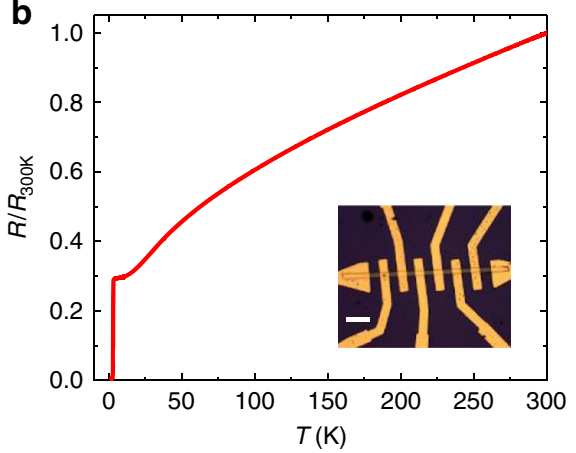

d

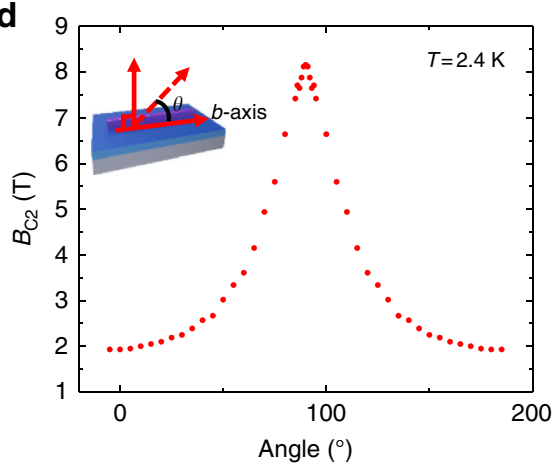

f

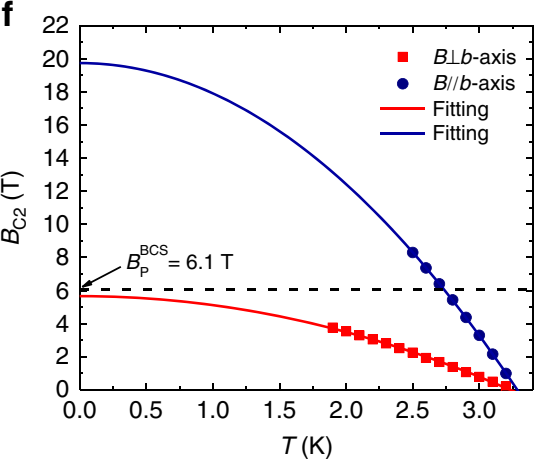

Fig. 2 Anisotropic superconducting behavior in $\mathrm{Ta}_{2} \mathrm{PdS}_{5}$ nanowire device. a Schematic device structure based on a $\mathrm{Ta}_{2} \mathrm{PdS}_{5}$ nanowire. b $\mathrm{Temperature}$ dependence of the normalized resistance (red solid line) of $\mathrm{Ta}_{2} \mathrm{PdS}_{5}$ nanowire device with a cross-sectional area of $120 \mathrm{~nm}$ (thickness) $\times 300 \mathrm{~nm}$ (width). Inset, an optical image of the $\mathrm{Ta}_{2} \mathrm{PdS}_{5}$ device, scale bar, $5 \mu \mathrm{m}$. c The temperature-dependent resistance of the device under a magnetic field perpendicular to the substrate, the resistance is normalized to the normal-state value right above the superconducting transition. $\mathbf{d}$ Angular dependence of the critical magnetic field $B_{\mathrm{C} 2}$ of a similar device measured at $2.4 \mathrm{~K}$. Inset, Schematic configuration of angular-dependent magnetoresistance measurement. The $\theta=0$ is defined as the magnetic field parallel to the $b$-axis. e Normalized $R-T$ characteristics of $\operatorname{Ta}_{2} \mathrm{PdS}_{5}$ under the magnetic field parallel to the $b$-axis.

f Temperature-dependent $B_{\mathrm{C} 2}$ for the magnetic field parallel and perpendicular to the $b$-axis. The red and blue solid lines are the theoretical fitting of $B_{\mathrm{C} 2}(T)$ $=B_{C 2}(0)\left[1-\left(T / T_{C}\right)^{2}\right]$. The black dashed line is the Pauli limit in response to the superconducting transition temperature

characteristics is a hybrid effect resulting from both self-heating hotspots and the runaway and retrapping of the phase point in the tilted washboard-like potential of the underdamped Josephson junction ${ }^{34}$. We have also compared the $I-V$ relation of $\mathrm{Ta}_{2} \mathrm{PdS}_{5}$ nanowires with thickness changes from $\sim 5 \mu \mathrm{m}$ (bulk) to $110 \mathrm{~nm}$ (See Supplementary Figure 9). For $\sim 5 \mu \mathrm{m}$ thick (Bulk) sample, there is only one step in the $I-V$ curves and the voltage is zero under low current bias until the current reaches a certain value $I_{C}$ (Note that the data points in Supplementary Figure 9 (a) at $V \sim 1 \mu V$ are the noise approaching the measurement limit). As the thickness of the device goes down, multiple voltage steps and enhanced Ohmic finite resistance emerge, which is mainly due to the $1 \mathrm{D}$ confinement effect as predicted by thermal-activated phase slip model ${ }^{32,35,36}$ (See Supplementary Note 4 for details). In addition, we have fit the temperaturedependent critical current to the Bardeen's formula ${ }^{37,38}$ for quasi-1D superconductors $I(T)=I_{C}(0)\left(1-\left(T / T_{C 0}\right)^{2}\right)^{3 / 2}$, where $T_{C 0}$ is the transition temperature $T$ in the absence of currents and fields (See Supplementary Figure 10a for details). We found that the experimental data fit the equation well, evidencing the quasi-1D superconductivity in $\mathrm{Ta}_{2} \mathrm{PdS}_{5}$ nanowires. Note that our observation of multi-steps and hysteresis in $I-V$ curves in $\mathrm{Ta}_{2} \mathrm{PdS}_{5}$ nanowires is consistent with the scenario generally expected in quasi-1D nanowires as well ${ }^{17}$. At first glance, it seems contrary to the fact that the GL coherence length of $\mathrm{Ta}_{2} \mathrm{PdS}_{5}$, as calculated above and described in Supplementary Note 3 , is much smaller than the sample size. However, it has been suggested that for type-II superconductors the required actual size for the quasi-1D superconductivity can be determined by their penetration depth ${ }^{17,38}$, unlike in type-I superconductors where the diameter of the wires needs to be less than its GL coherence length. 

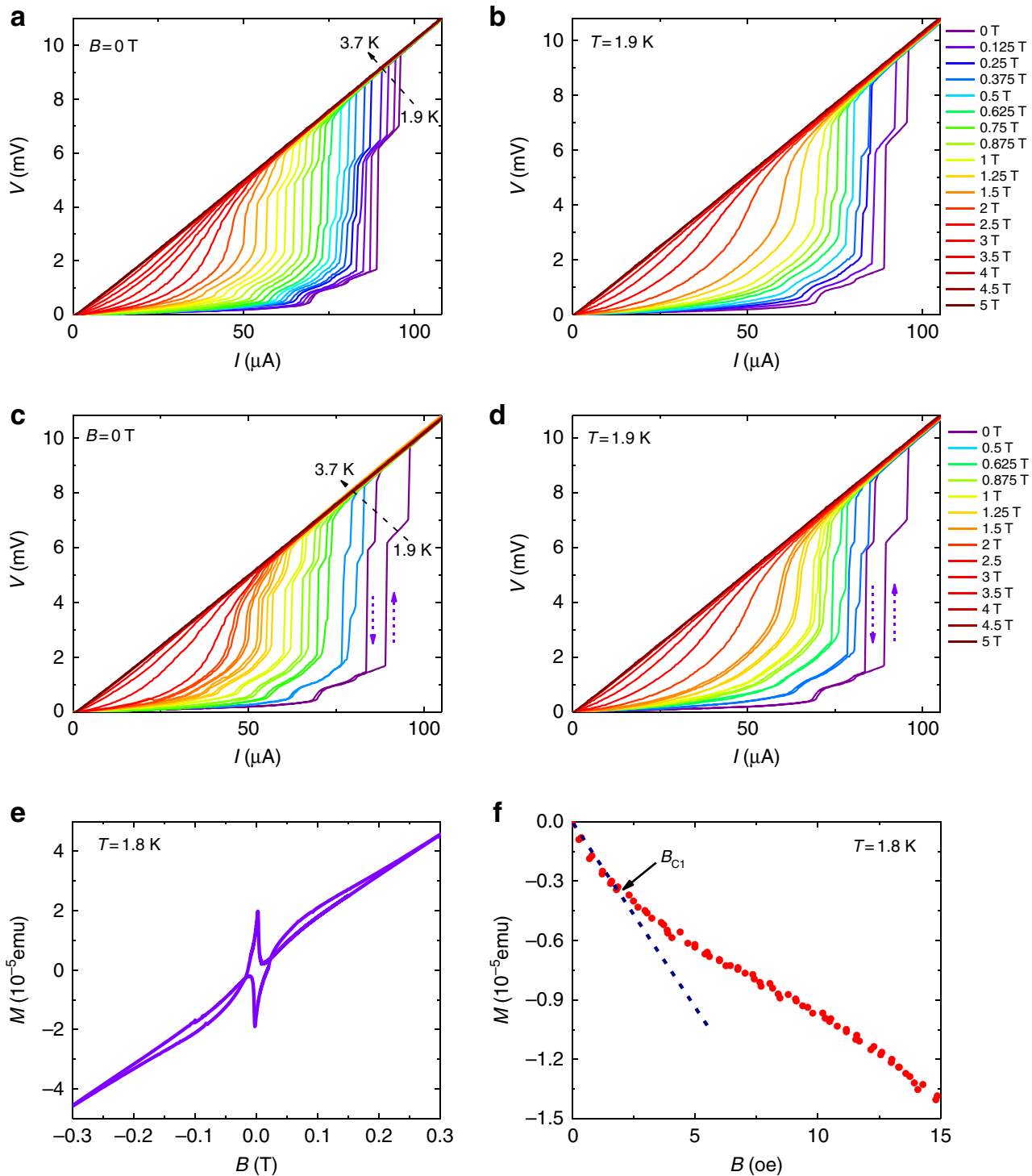

Fig. $3 \mathrm{I}-\mathrm{V}$ characteristics and magnetic properties of $\mathrm{Ta}_{2} \mathrm{PdS}_{5}$. a Temperature-dependent $\mathrm{I}-\mathrm{V}$ curves of the $\mathrm{Ta}_{2} \mathrm{PdS}_{5}$ nanowire in the linear scale under increased current bias, showing multiple voltage steps in the superconducting transition regime. $\mathbf{b} I-V$ curves of the $\operatorname{Ta}_{2} \mathrm{PdS}_{5} \mathrm{nanowire} \mathrm{under}_{\mathrm{sarious}}$ magnetic fields perpendicular to the substrate, in which multiple voltage steps were also observed under various magnetic fields. c, $\mathbf{d}$ Hysteresis loops in $/$ $V$ curves when the current sweeps up and down at different temperatures and perpendicular magnetic fields, respectively. e Magnetic moment as a function of magnetic field in $\mathrm{Ta}_{2} \mathrm{PdS}_{5}$, which shows a typical behavior of type-II superconductor. $\mathbf{f}$ Expanded region of the initial magnetization curve up to $15 \mathrm{Oe}$ in $\mathrm{Ta}_{2} \mathrm{PdS}_{5}$, where $B_{\mathrm{C} 1}(1.8 \mathrm{~K})=1.8 \mathrm{Oe}$ can be extracted

We then extract the magnetic properties of $\mathrm{Ta}_{2} \mathrm{PdS}_{5}$ to estimate the penetration depth. The obtained magnetic moment versus magnetic field of $\mathrm{Ta}_{2} \mathrm{PdS}_{5}$ is shown in Fig. 3e, exhibiting a typical behavior of type-II superconductors ${ }^{39,40}$. From the initial magnetization curve (Fig. $3 \mathrm{f}), B_{\mathrm{C} 1}(1.8 \mathrm{~K})=1.8$ Oe can be acquired. Using the equation ${ }^{20} B_{\mathrm{C}}(T)=B_{\mathrm{C}}(0)\left[1-\left(T / T_{\mathrm{C}}\right)^{2}\right]$, we can obtain the low temperature $B_{\mathrm{Cl}}(0)=2.6$ Oe. Utilizing the $B_{\mathrm{C} 2 \mathrm{a}^{\circ}}(0)=4.7 \mathrm{~T}$ in Supplementary Note $3^{39}$, the GL parameter can be estimated to be 220 following the equation 20,25 $B_{C 2}(0) / B_{C 1}(0)=2 \kappa(0)^{2} / \ln \kappa(0)$. Using ${ }^{20,25} \kappa(0)=\lambda(0) / \xi(0)$, we can obtain the penetration depth $\lambda(0)$ of $\mathrm{Ta}_{2} \mathrm{PdS}_{5}$ nanowire $\sim 1848 \mathrm{~nm}$, which is much larger than the device thickness of $120 \mathrm{~nm}$. Note that the penetration depth we estimated here is similar to other quasi-1D superconductors like $\mathrm{Nb}_{2} \mathrm{PdS}_{5}$ $\sim 785 \mathrm{~nm}$ [ref. ${ }^{41}$ ], $\mathrm{Nb}_{2} \mathrm{PdS}_{5} \sim 410 \mathrm{~nm}$ [ref. ${ }^{20}$ ] and $\mathrm{Sc}_{3} \mathrm{CoC}_{4}$ $\sim 970 \mathrm{~nm}$ [ref. ${ }^{39}$ ]. Thus, we believe that the superconductivity in $\mathrm{Ta}_{2} \mathrm{PdS}_{5}$ is quasi- $1 \mathrm{D}$.
Superconductor-metal transition and quantum Griffiths state. Next, we study the magnetoresistance of the $\mathrm{Ta}_{2} \mathrm{PdS}_{5}$ in a perpendicular magnetic field configuration. Figure $4 \mathrm{a}$ reveals the temperature-dependent resistance of a nanowire device (crosssectional area $300 \mathrm{~nm} \times 100 \mathrm{~nm}$ ) under various magnetic fields. With the increase of the magnetic field, the superconducting transition shifts monotonically to lower temperatures similar to Fig. 2c. As the magnetic field continues to increase, the superconductor gradually changes to a localized metal, indicating a magnetic field induced SMT. This SMT behavior is further explored in magnetoresistance isotherms in Fig. $4 \mathrm{~b}$ where $R_{\mathrm{S}}(B)$ curves cross each other. Intriguingly, the crossing point of SMT seemingly changes as the temperature varies. To investigate the SMT behavior in $\mathrm{Ta}_{2} \mathrm{PdS}_{5}$ nanowires, we measure the sample in the dilution temperature environment. Figure $4 \mathrm{c}$ shows the magnetoresistance isotherms of the device under an ultra-low temperature ranging from 0.12 to $1.2 \mathrm{~K}$. A series of crossing 

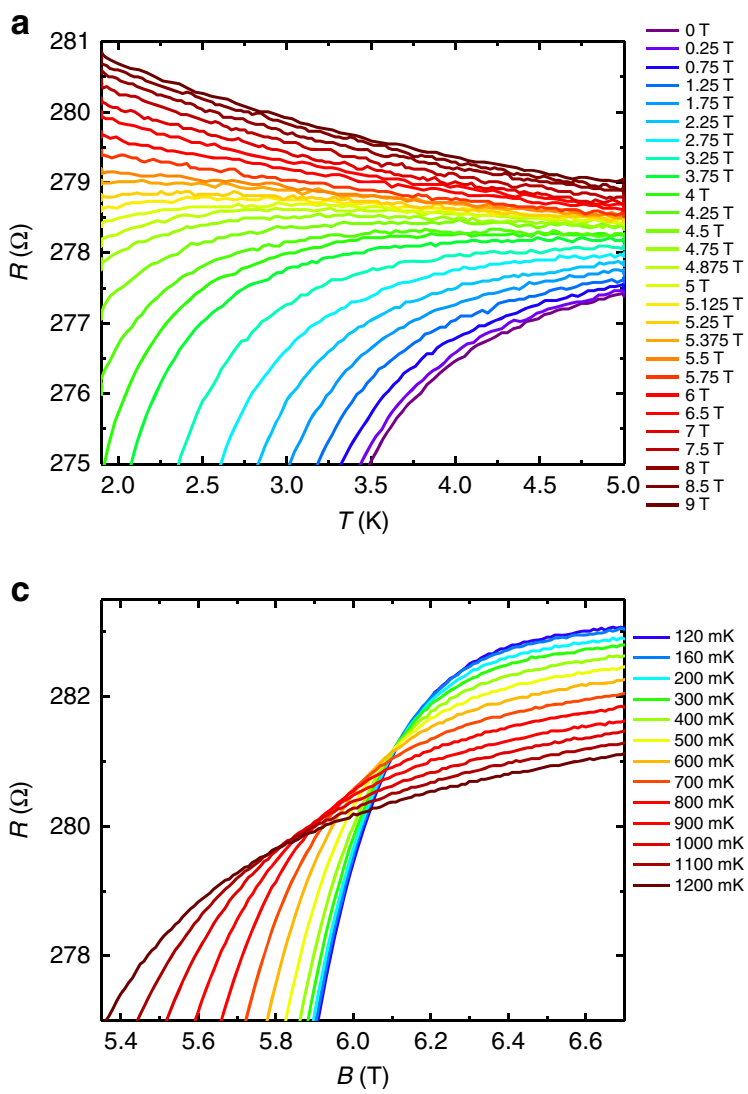

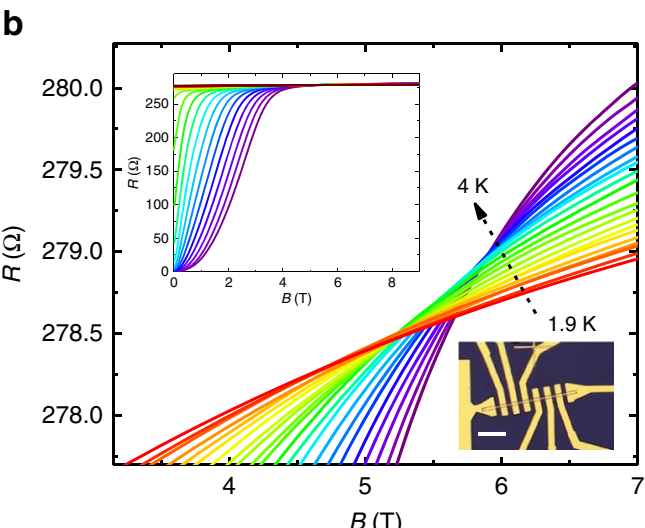

d

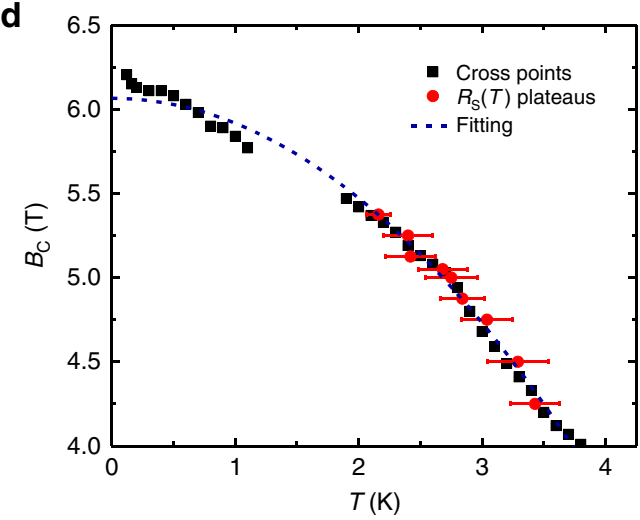

Fig. 4 Superconducting-metal transition in $\mathrm{Ta}_{2} \mathrm{PdS}_{5}$ nanowire device with the thickness of $100 \mathrm{~nm}$. a Zoom-in view of the temperature-dependent resistance at perpendicular magnetic field ranging from 0 to $9 \mathrm{~T}$, showing an insulating behavior at high magnetic fields. b Zoomed magnetoresistance of the device measured at a temperature of 1.9-4 K under perpendicular magnetic field configuration, showing a series of cross points as temperature changes. Inset: up left, the whole range of the magnetoresistance isotherms; down right, an optical image of the device, scale bar $10 \mu \mathrm{m}$. $\mathbf{c}$ Perpendicular magnetoresistance isotherms of the device at various temperatures ranging from 0.12 to $1.2 \mathrm{~K}$. $\mathbf{d}$ Critical magnetic fields $B_{C}(T)$ extracted from $\mathbf{a}$, $\mathbf{b}$, and $\mathbf{c}$. Black squares are the crossing points of $R_{S}(B)$ curves at every two adjacent temperatures around the transition regime. The red dots are taken from the $R_{\mathrm{S}}(T)$ curves at given magnetic fields in a where $\mathrm{d} R_{\mathrm{S}} / \mathrm{d} T$ changes sign. Error bars of the red dots represent the temperature uncertainty due to the experimental resolution in a where $\mathrm{d} R_{\mathrm{S}} / \mathrm{d} T$ changes sign. The blue dashed line is the theoretical fitting to $B_{\mathrm{C} 2}(T)=B_{\mathrm{C} 2}(0)\left[1-\left(T / T_{\mathrm{C}}\right)^{2}\right]$

points have also been observed. We summarize the crossing points in both high and low-temperature regimes in Fig. 4d, where the black squares are crossing points of every two adjacent $R_{\mathrm{S}}(B)$ curves. Also, as shown by the red dots in Fig. $4 \mathrm{~d}$, the $R_{\mathrm{S}}$ plateaus where $\mathrm{d} R_{\mathrm{S}}(T) / \mathrm{d} T$ changes sign for a given magnetic field have been extracted from $R_{\mathrm{S}}(T)$ curves in Fig. 4a. We have also used the empirical equation to fit the crossing points (blue dashed line in Fig. 4d). In the high-temperature regime, the data fit the equation well; while in the ultra-low-temperature region, $B_{\mathrm{C}}$ diverges from the tendency of the empirical equation $(T<0.7 \mathrm{~K})$. The experimental data of $B_{C}(0)>6.2 \mathrm{~T}$ is substantially larger than the fitted result from the empirical equation $\left(B_{\mathrm{C}}(0)=6.06 \mathrm{~T}\right)$.

Typically, for SIT, the critical resistance corresponding to the border between the superconducting and insulating region at low temperatures should be the quantum unit of resistance $\left(\mathrm{h} / 4 \mathrm{e}^{2} \sim\right.$ $6450 \Omega$ ), and the critical exponent remains to be a constant ${ }^{42}$. Theoretical investigations $s^{43}$ show that unpaired electrons could originate from the pair-breaking mechanism of dissipation effect, giving rise to a much smaller critical resistance than $h / 4 \mathrm{e}^{2}$. This explains our experimental data that the critical resistance of $\mathrm{Ta}_{2} \mathrm{PdS}_{5}$ nanowire is much smaller than the quantum resistance $\mathrm{h} / 4 \mathrm{e}^{2}$. More recently, theoretical studies also show that quenched disorders could dramatically change the scaling behavior of SMT and result in an activated scaling behavior identical to that of the quantum random transverse-field Ising model ${ }^{44}$. In that system, the activated scaling behavior, called quantum Griffiths singularity, exhibits continuously varying dynamical exponent $z$ when approaching the infinite-randomness quantum critical point. Recent experiments on 2D superconducting Ga thin film, monolayer $\mathrm{NbSe}_{2}$ and ionic liquid gated $\mathrm{ZrNCl}$ and $\mathrm{MoS}_{2}$ have envisaged the similar continuous line of "critical" points and the divergence of the dynamical critical exponent in SMT, which experimentally reveals the existence of quantum Griffiths singularity state in SMT in 2D superconductors ${ }^{12,14}$.

Inspired by the new discovery of quantum Griffiths singularity in SMT, we analyze multiple crossing points in $\mathrm{Ta}_{2} \mathrm{PdS}_{5}$ nanowires using the scaling analysis method, according to which the resistance in the vicinity of a critical point follows ${ }^{, 45}$

$$
R(\delta, T)=R_{\mathrm{C}} f\left(\delta T^{-1 / z v}\right),
$$

where the $\delta=\left|B-B_{\mathrm{C}}\right|$ is the deviation from the critical field, $R_{\mathrm{C}}$ is the critical resistance, $f(x)$ is the scaling function with $f(0)=1, \delta$ is tuning parameter, $z$ is the dynamic critical exponent, and $v$ is the correlation length critical exponent. One "critical" point $\left(B_{\mathrm{C}}, R_{\mathrm{C}}\right)$ of a certain small critical transition region is defined as the crossing points of several adjacent $R_{\mathrm{S}}(B)$ curves (See Supplementary Figure 11 for details). The deduced magnetic field dependence of the effective "critical" exponent $z v$ is shown in Fig. 5. In the relative high-temperature regime, $z v$ value increases 


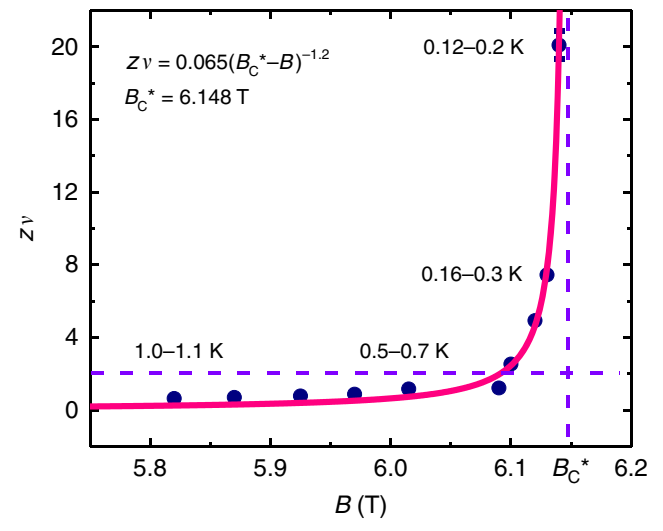

Fig. 5 The activated quantum scaling behavior in $\mathrm{Ta}_{2} \mathrm{PdS}$. Deduced exponent $z \nu$ as a function of magnetic field, showing no sign of saturation when approaching the zero-temperature limit. The solid pink line is the fit based on the activated scaling law equation as shown in the figure, two violet dashed lines represent the constant values with $B_{C}{ }^{*}=6.148 \mathrm{~T}$ and $z \nu$ $=2$. The error bar representing the width of $z v$ value was acquired during the scaling analysis performed in Supplementary Figure 11

slowly with the magnetic field, while in the ultra-low-temperature regime, $z v$ grows quickly as the temperature decreases. Here we note that one $z v$ value corresponds to a temperature region rather than a certain temperature. Next, we try to fit the extracted $z v$ values versus $\mathrm{B}$ using the activated scaling law $z v=C \mid B_{C^{*}}-$ $\left.B\right|^{-\eta \psi}$, where $C$ is a constant, $\eta \approx 1.2$ is the correlation length exponent and $\psi \approx 1$ is the tunneling critical exponent in quasione dimension ${ }^{12,14,46}$. The activated scaling law fits the data well (the solid pink line in Fig. 5, where $B_{\mathrm{C}}{ }^{*}=6.148 \mathrm{~T}$ ), indicating the existence of infinite-randomness quantum critical points in $\mathrm{Ta}_{2} \mathrm{PdS}_{5}$ nanowires. As mentioned before, the enhanced quenched disorders ${ }^{13}$ at low temperatures are the main inducement of the quantum Griffiths singularity in $\mathrm{Ta}_{2} \mathrm{PdS}_{5}$. Although the $\mathrm{Ta}_{2} \mathrm{PdS}_{5}$ nanowires are single crystalline with good uniformity, there may still be some defects. In addition, the interface effect coming from the device fabrication and scattering from the substrate could also introduce disorders in the system. All of these above could be the origin of the enhanced quenched disorders at low temperatures ${ }^{14}$, resulting in the infinite $z v$ value when the critical point $B_{C}{ }^{*}$ is approached.

\section{Discussion}

With the reduced dimensionality of angle-dependent $B_{\mathrm{C} 2}$ of thin $\mathrm{Ta}_{2} \mathrm{PdS}_{5}$ nanowires, the appearance of multiple $I-V$ steps, the enhanced Ohmic finite resistance when the thickness of the device reduces, the good fittings of the temperature-dependent critical current to the Bardeen's formula ${ }^{37,38}$ for quasi-1D superconductors, and the $z v$ value to the $1 \mathrm{D}$ equation ${ }^{46} z v=$ $C\left|B_{C^{*}}-B\right|^{-1.2}$, the quasi-1D superconductivity is suggested to present in $\mathrm{Ta}_{2} \mathrm{PdS}_{5}$ nanowires. Considering the crystal lattice constants $^{19} a=1.2 \mathrm{~nm}, b=0.3 \mathrm{~nm}$, and $c=1.5 \mathrm{~nm}$, the GL coherence lengths along different crystal axes are larger than the lattice constants $\left(\xi_{\mathrm{a}} \cdot(0)=8.4 \mathrm{~nm}, \xi_{\mathrm{b}}(0)=4.2 \mathrm{~nm}\right.$, and $\xi_{\mathrm{c}} \cdot(0)=8.6$ $\mathrm{nm}$, see Supplementary Note 3 for details). Note that the GL coherence length along different directions is also smaller than our nanowire thickness $(\sim 120 \mathrm{~nm})$. However, the actual effective superconducting thickness of $\mathrm{Ta}_{2} \mathrm{PdS}_{5}$ nanowire should be smaller than the sample thickness ${ }^{3}$, which may make the superconductivity in $\mathrm{Ta}_{2} \mathrm{PdS}_{5}$ nanowires quasi-1D. Nevertheless, the penetration depth of $\sim 1848 \mathrm{~nm}$ calculated above is significantly larger than the sample thickness, satisfying the condition required for quasi-1D superconductivity in type-II superconductors ${ }^{17,38}$.
Also, from previous experiments on quasi-1D superconducting nanowires, the diameter of the systems ranges from 10 to 1000 $\mathrm{nm}^{32,34,38,47-49}$ and our sample thickness is within that range. We need to further mention that the unique weak coupled Ta-S chains ${ }^{19,27,50}$ along the $b$-axis, which are responsible for the superconductivity also make the superconductivity in $\mathrm{Ta}_{2} \mathrm{PdS}_{5}$ nanowires quasi-1D $\mathrm{D}^{39,51}$. Considering all the facts listed above, we conclude that the superconductivity in $\mathrm{Ta}_{2} \mathrm{PdS}_{5}$ nanowire is quasi-1D in nature (also see Supplementary Note 5 for all listed facts for quasi-1D superconductivity in $\mathrm{Ta}_{2} \mathrm{PdS}_{5}$ nanowires).

The observation of quantum Griffiths singularity state in $\mathrm{Ta}_{2} \mathrm{PdS}_{5}$ also suggests that the critical phenomenon below the quantum critical point is described by exponentially small but nonzero probability of large-ordered regions-rare regions, which can be viewed as the superconducting puddles surviving in the normal state background with a long time and length scale as the temperature approaches $0 \mathrm{~K}^{15,52}$. Instead of one or two crossing points in the magnetoresistance isotherms cross in the SIT or SMT, recent observations of the quantum Griffiths singularity in thin $\mathrm{Ga}^{12}$ films and monolayer $\mathrm{NbSe}_{2}{ }^{14}$ have shown one cross point in the high-temperature regime and a series of crossing points in the ultra-low-temperature regime. Interestingly, our study in quasi-1D $\mathrm{Ta}_{2} \mathrm{PdS}_{5}$ nanowire has shown that the crossing point moves continuously in both high- and low-temperature regimes. As a result, the dynamical exponent $z$ continuously varies in both high- and low-temperature regimes. The reason for this may be due to the fact that the thermal fluctuations at such a temperature regime $(2-4 \mathrm{~K})$ are unable to smear out the inhomogeneity caused by quenched disorders $12,53,54$. However, the exquisite physics in this system needs further theoretical and experimental investigations.

In summary, we have demonstrated the systematic study of magnetotransport properties of quasi-1D $\mathrm{Ta}_{2} \mathrm{PdS}_{5}$ nanowires. The strong anisotropic superconducting behavior and hysteretic multiple voltage steps in its $I-V$ relation indicate its typical quasi$1 \mathrm{D}$ nature in superconductivity. Importantly, the nanowire undergoes a SMT and shows signatures of quantum Griffiths singularity state when approaching zero-temperate quantum critical point. These findings shed lights on the understanding of the superconductor-metal and metal-insulator transitions. In addition, the appealing physical properties unveiled in this study demonstrate $\mathrm{Ta}_{2} \mathrm{PdS}_{5}$ to be a promising platform for possible applications in quantum computing devices.

\section{Methods}

Sample preparation. Single crystals of $\mathrm{Ta}_{2} \mathrm{PdS}_{5}$ were synthesized by the CVT method using iodine as a transport agent. Before the crystal growth, a quartz tube containing iodine and the stoichiometric ratio of $\mathrm{Ta}, \mathrm{Pd}$, and $\mathrm{S}$ powders with $1 \%$ excess of $S$ was evacuated and sealed. The sealed tube was then placed in a double zone furnace horizontally and grew for 2 weeks with the temperature gradient of $775-850^{\circ} \mathrm{C}$, after which needle-like single crystals of $\mathrm{Ta}_{2} \mathrm{PdS}_{5}$ were formed at the low-temperature end.

Material characterizations. The structural and compositional characteristics of the nanowires were investigated using TEM (FEI Tecnai F20, $200 \mathrm{kV}$, equipped with EDS). The nanowires were dry transferred onto Lacey carbon films supported by a copper grid.

Device fabrication. $\mathrm{Ta}_{2} \mathrm{PdS}_{5}$ nanowires with different thickness were obtained through mechanical exfoliation onto pre-patterned $\mathrm{SiO}_{2}(285 \mathrm{~nm}) / \mathrm{Si}$ substrates from bulk crystals. The electrical contacts of $\mathrm{Ta}_{2} \mathrm{PdS}_{5}$ devices were fabricated along the $b$-axis of the crystal by EBL using Polymethylmethacrylate/Methyl methacrylate bilayer polymer. Ti/Au $(5 \mathrm{~nm} / 150 \mathrm{~nm})$ electrodes were then deposited using magnetron sputtering.

Transport measurements. Four-terminal temperature-dependent magnetotransport and $I-V$ measurement measurements were carried out in a Physical Property Measurement System (PPMS) system (Quantum Design) using lock-in amplifier (SR830), Agilent 2912 and Keithley 2182. Ultra-low-temperature magnetotransport 
measurements were performed in an Oxford $3 \mathrm{He} / 4 \mathrm{He}$-dilution refrigerator equipped with a superconducting magnet using SR830.

\section{Data availability}

All of the experimental data supporting this study are available from the corresponding author.

Received: 2 April 2018 Accepted: 18 October 2018

Published online: 07 November 2018

\section{References}

1. Saito, Y., Nojima, T. \& Iwasa, Y. Highly crystalline 2D superconductors. Nat. Rev. Mater. 2, 16094 (2016).

2. Iwasa, Y., Saito, Y., Kasahara, Y., Ye, J. \& Nojima, T. Metallic ground state in an ion-gated two-dimensional superconductor. Science 350, 409-413 (2015).

3. $\mathrm{Xu}, \mathrm{C}$. et al. Large-area high-quality $2 \mathrm{D}$ ultrathin $\mathrm{Mo}_{2} \mathrm{C}$ superconducting crystals. Nat. Mater. 14, 1135-1141 (2015).

4. Mooij J. in Percolation, Localization, and Superconductivity. NATO Science Series, vol. 109 (eds Goldman, A.M. \& Wolf, S.A.) 325-370 (Plenum Press, New York, 1984)

5. Guo, Y. et al. Superconductivity modulated by quantum size effects. Science 306, 1915-1917 (2004)

6. Orr, B., Jaeger, H. \& Goldman, A. Transition-temperature oscillations in thin superconducting films. Phys. Rev. Lett. 53, 2046 (1984).

7. Bollinger, A. T. et al. Superconductor-insulator transition in $\mathrm{La}_{2-\mathrm{x}} \mathrm{Sr}_{\mathrm{x}} \mathrm{CuO}_{4}$ at the pair quantum resistance. Nature 472, 458-460 (2011).

8. Fisher, M. P. Quantum phase transitions in disordered two-dimensional superconductors. Phys. Rev. Lett. 65, 923 (1990).

9. Goldman, A. M. \& Markovic, N. Superconductor-insulator transitions in the two-dimensional limit. Phys. Today 51, 39-44 (1998).

10. Liu, Y., Haviland, D., Nease, B. \& Goldman, A. Insulator-to-superconductor transition in ultrathin films. Phys. Rev. B 47, 5931 (1993).

11. Tsen, A. W. et al. Nature of the quantum metal in a two-dimensional crystalline superconductor. Nat. Phys. 12, 208-212 (2015).

12. Xing, Y. et al. Quantum Griffiths singularity of superconductor-metal transition in Ga thin films. Science 350, 542-545 (2015).

13. Shen, S. et al. Observation of quantum Griffiths singularity and ferromagnetism at the superconducting $\mathrm{LaAlO}_{3} / \mathrm{SrTiO}_{3}(110)$ interface. Phys. Rev. B 94, 144517 (2016).

14. Xing, Y. et al. Ising Superconductivity and quantum phase transition in macro-size monolayer $\mathrm{NbSe}_{2}$. Nano. Lett. 17, 6802-6807 (2017).

15. Saito, Y., Nojima, T. \& Iwasa, Y. Quantum phase transitions in highly crystalline two-dimensional superconductors. Nat. Commun. 9, 778 (2018).

16. Markovic, N. Randomness rules. Science 350, 509-509 (2015).

17. Ning, W. et al. Superconductor-insulator transition in quasi-onedimensional single-crystal $\mathrm{Nb}_{2} \mathrm{PdS}_{5}$ nanowires. Nano. Lett. 15, 869-875 (2015).

18. Jha, R., Tiwari, B., Rani, P., Kishan, H. \& Awana, V. P. S. Robust superconductivity with large upper critical field in $\mathrm{Nb}_{2} \mathrm{PdS}_{5}$. J. Appl. Phys. 115, 213903 (2014)

19. Lu, Y. et al. Superconductivity at $6 \mathrm{~K}$ and the violation of Pauli limit in $\mathrm{Ta}_{2} \mathrm{Pd}_{\mathrm{x}} \mathrm{S}_{5}$. J. Phys. Soc. Jpn. 83, 023702 (2014).

20. $\mathrm{Yu}, \mathrm{H}$. et al. Superconducting fiber with transition temperature up to $7.43 \mathrm{~K}$ in $\mathrm{Nb}_{2} \mathrm{PdxS}_{5}$-delta $(0.6<\mathrm{x}<1)$. J. Am. Chem. Soc. 135, 12987-12989 (2013).

21. Singh, D. J. Electronic structure and upper critical field of superconducting $\mathrm{Ta}_{2} \mathrm{PdS}_{5}$. Phys. Rev. B 88, 174508 (2013).

22. Squattrito, P. J., Sunshine, S. A. \& Ibers, J. A. Some substitutional chemistry in known ternary and quaternary chalcogenides. J. Solid State Chem. 64, 261-269 (1986).

23. Qin, F. et al. Superconductivity in a chiral nanotube. Nat. Commun. 8, 14465 (2017).

24. Zeng, J. et al. Gate-Induced Interfacial Superconductivity in $1 \mathrm{~T}-\mathrm{SnSe}_{2}$. Nano. Lett. 18, 1410-1415 (2018).

25. Tinkham M. Introduction to Superconductivity. (Courier Corporation, New York, 2004).

26. $\mathrm{Lu}, \mathrm{J}$. M. et al. Evidence for two-dimensional Ising superconductivity in gated $\mathrm{MoS}_{2}$. Science 350, 1353-1357 (2015).

27. Khim, S. et al. Enhanced upper critical fields in a new quasi-one-dimensional superconductor $\mathrm{Nb}_{2} \mathrm{PdxSe}_{5}$. New J. Phys. 15, 123031 (2013).

28. $\mathrm{Xi}, \mathrm{X}$. et al. Ising pairing in superconducting $\mathrm{NbSe}_{2}$ atomic layers. Nat. Phys. 12, 139-143 (2015)

29. Zhang, Q. et al. Superconductivity with extremely large upper critical fields in $\mathrm{Nb}_{2} \mathrm{Pd}_{0.81} \mathrm{~S}_{5}$. Sci. Rep. 3, 1446 (2013).
30. Xu, K. \& Heath, J. R. Controlled fabrication and electrical properties of long quasi-one-dimensional superconducting nanowire arrays. Nano. Lett. 8 , 136-141 (2008)

31. Rogachev, A. \& Bezryadin, A. Superconducting properties of polycrystalline $\mathrm{Nb}$ nanowires templated by carbon nanotubes. Appl. Phys. Lett. 83, 512-514 (2003).

32. Tian, M. et al. Dissipation in quasi-one-dimensional superconducting singlecrystal Sn nanowires. Phys. Rev. B 71, 104521 (2005).

33. Tinkham, M., Free, J. U., Lau, C. N. \& Markovic, N. Hysteretic I-V curves of superconducting nanowires. Phys. Rev. B 68, 134515 (2003).

34. $\mathrm{Li}, \mathrm{P}$. et al. Switching currents limited by single phase slips in one-dimensional superconducting Al nanowires. Phys. Rev. Lett. 107, 137004 (2011).

35. Lukens, J. E., Warburton, R. J. \& Webb, W. W. Onset of quantized thermal fluctuations in "one-dimensional" superconductors. Phys. Rev. Lett. 25, $1180-1184(1970)$

36. Newbower, R., Beasley, M. \& Tinkham, M. Fluctuation effects on the superconducting transition of tin whisker crystals. Phys. Rev. B 5, 864 (1972).

37. Damaschke, B., Yang, X. \& Tidecks, R. The hysteresis of the critical current in superconducting tin whiskers. J. Low. Temp. Phys. 70, 131-150 (1988).

38. Tidecks R. Current-Induced Nonequilibrium Phenomena in Quasi-OneDimensional Superconductors. (Springer-Verlag, Berlin, Heidelberg GmbH, 2006).

39. Scheidt, E. W., Hauf, C., Reiner, F., Eickerling, G. \& Scherer, W. Possible indicators for low dimensional superconductivity in the quasi-1D carbide $\mathrm{Sc}_{3} \mathrm{CoC}_{4}$. J. Phys.: Conf. Ser. 273, 012083 (2011).

40. Senoussi, S., Oussena, M. \& Hadjoudj, S. On the critical fields and current densities of $\mathrm{YBa}_{2} \mathrm{Cu}_{3} \mathrm{O}_{7}$ and $\mathrm{La}_{1.85} \mathrm{Sr}_{0.15} \mathrm{CuO}_{4}$ superconductors. J. Appl. Phys. 63, 4176-4178 (1988).

41. Biswas, P. K. et al. Nodeless superconductivity in quasi-one-dimensional $\mathrm{Nb}_{2} \mathrm{PdS}_{5}$ : A $\mu$ SR study. Phys. Rev. B 91, 100504 (2015).

42. Hebard, A. \& Paalanen, M. Magnetic-field-tuned superconductor-insulator transition in two-dimensional films. Phys. Rev. Lett. 65, 927 (1990).

43. Kapitulnik, A., Mason, N., Kivelson, S. A. \& Chakravarty, S. Effects of dissipation on quantum phase transitions. Phys. Rev. B 63, 125322 (2001).

44. Fisher, D. S. Critical behavior of random transverse-field Ising spin chains. Phys. Rev. B 51, 6411 (1995).

45. Sondhi, S., Girvin, S., Carini, J. \& Shahar, D. Continuous quantum phase transitions. Rev. Mod. Phys. 69, 315 (1997).

46. Motrunich, O., Mau, S.-C., Huse, D. A. \& Fisher, D. S. Infinite-randomness quantum Ising critical fixed points. Phys. Rev. B 61, 1160 (2000).

47. Werner, T., Tidecks, R. \& Johnston, B. Whiskers from the In-Pb system: growth, handling and characteristic properties. J. Cryst. Growth 73, 467-481 (1985).

48. Schulz, U. \& Tidecks, R. Dissipative state of superconducting zinc whiskers. $J$. Low. Temp. Phys. 71, 151-171 (1988).

49. Slama, G. \& Tidecks, R. The influence of the electron mean free path on phase-slip centers in indium whiskers. Solid State Commun. 44, 425-429 (1982).

50. Zhang, J. et al. Superconductivity at $2.5 \mathrm{~K}$ in the new transition-metal chalcogenide $\mathrm{Ta}_{2} \mathrm{PdSe}_{5}$. Supercond. Sci. Technol. 28, 115015 (2015).

51. Bergk, B. et al. Superconducting transitions of intrinsic arrays of weakly coupled one-dimensional superconducting chains: the case of the extreme quasi-1D superconductor $\mathrm{Tl}_{2} \mathrm{Mo}_{6} \mathrm{Se}_{6}$. New J. Phys. 13, 103018 (2011).

52. Spivak, B., Oreto, P. \& Kivelson, S. A. Theory of quantum metal to superconductor transitions in highly conducting systems. Phys. Rev. B 77, 214523 (2008).

53. Biscaras, J. et al. Multiple quantum criticality in a two-dimensional superconductor. Nat. Mater. 12, 542-548 (2013).

54. Shi, X., Lin, P. V., Sasagawa, T., Dobrosavljević, V. \& Popović, D. Two-stage magnetic-field-tuned superconductor-insulator transition in underdoped $\mathrm{La}_{2}$ ${ }_{x} \mathrm{Sr}_{\mathrm{x}} \mathrm{CuO}_{4}$. Nat. Phys. 10, 437-443 (2014).

\section{Acknowledgements}

This work was supported by the National Key Research and Development Program of China (grant no. 2018YFA0305601 and 2017YFA0303302) and National Natural Science Foundation of China (11474058, 61674040, and 11874116), and the Australian Research Council. Part of the sample fabrication was performed at Fudan Nano-fabrication Laboratory. The single-crystal growth at Tulane was supported by the US Department of Energy (DOE) under grant no. DE-SC0014208. We thank Jiwei Ling and Prof. Haiwen Liu for helpful discussions.

\section{Author contributions}

F.X. conceived the idea and supervised the experiments. E.Z., Z.F., L.A., J.S., C.H., S.L., Z.L., and X.Z. carried out the device fabrication. E.Z. performed low-temperature measurements and data analysis. J.Z., E.Z., N.K., H.X., W.W., and L.H. conducted the magnetotransport measurements at dilution temperature. J.L. and Z.M. synthetized 
$\mathrm{Ta}_{2} \mathrm{PdS}_{5}$ single crystals. Y-C.Z. and J.Z. performed structural characterization and analysis. E.Z. and F.X. wrote the paper with the help from all other authors.

\section{Additional information}

Supplementary Information accompanies this paper at https://doi.org/10.1038/s41467018-07123-y.

Competing interests: The authors declare no competing interests.

Reprints and permission information is available online at http://npg.nature.com/ reprintsandpermissions/

Publisher's note: Springer Nature remains neutral with regard to jurisdictional claims in published maps and institutional affiliations. (c) (i) Open Access This article is licensed under a Creative Commons Attribution 4.0 International License, which permits use, sharing, adaptation, distribution and reproduction in any medium or format, as long as you give appropriate credit to the original author(s) and the source, provide a link to the Creative Commons license, and indicate if changes were made. The images or other third party material in this article are included in the article's Creative Commons license, unless indicated otherwise in a credit line to the material. If material is not included in the article's Creative Commons license and your intended use is not permitted by statutory regulation or exceeds the permitted use, you will need to obtain permission directly from the copyright holder. To view a copy of this license, visit http://creativecommons.org/ licenses/by/4.0/.

(C) The Author(s) 2018 